Elsevier Editorial System(tm) for Nanomedicine: Nanotechnology, Biology, and

Medicine

Manuscript Draft

Manuscript Number: JN200967

Title: Self-assembled Multifunctional Fe/MgO Nanospheres for Magnetic Resonance Imaging and Hyperthermia

Article Type: New Technology

Keywords: nanoparticles; magnetism; hyperthermia; MRI

Corresponding Author: Mr Carlos Martinez Boubeta, Ph.D

Corresponding Author's Institution: In2UB

First Author: Carlos Martinez Boubeta, Ph.D

Order of Authors: Carlos Martinez Boubeta, Ph.D; Lluis Balcells; Rosa Cristòfol ; Coral Sanfeliu ; Elisenda Rodrígue; Ralph Weissleder ; Silvia Lope-Piedrafita; Konstantinos Simeonidis; Makis Angelakeris; Felip Sandiumenge; Alberto Calleja; Lluis Casas; Claude Monty; Benjamin Martínez

Abstract: A one-step process for the production of nanoparticles presenting advanced magnetic properties can be achieved using vapor condensation. In this paper, we report on the fabrication of Fe particles covered by a uniform MgO epitaxial shell. MgO has a lower surface energy than Fe, which results in a core-shell crystal formation. The particles are proven to be useful as as contrast agents for magnetic resonance diagnosis and heating mediators for cancer therapy through hyperthermia. They also have potential to be used in drug delivery and magnetic-activated cell sorting. 
Dear Editor,

Enclosed is the paper "Self-assembled Multifunctional Fe/MgO Nanospheres for Magnetic Resonance Imaging and Hyperthermia" by Carlos Martínez-Boubeta et al. for your consideration as a potential contribution in the NanoMedicine. The manuscript, or any part of it, is not and will not be submitted elsewhere for publication while under consideration by Nanomedicine. All authors have seen and approved the submission of this manuscript.

While a huge number of methods have been developed for the scalable synthesis and preparation of inorganic metal and semiconductor nanoparticles, only a few specialized techniques were reported for the preparation of coreshell nanoparticles. In this paper, we reported a novel and versatile technique for the preparation of injectable nanoparticle dispersions suitable to act as contrast agents for magnetic resonance imaging. Our ferromagnetic particles (and not superparamagnetic, as widely reported) embedded in a MgO shell may eventually be useful to diagnose and treat human diseases by means of hyperthermia. Due to their special magnetic and electronic properties and simple solution processing, such dispersions of nanoparticles are promising materials for future applications in electronics and optical (bio)-sensors. Worth of mention, as archetypical technology materials, Fe-MgO particles and films have been intensively studied because they possess a variety of outstanding physicochemical properties which are utilized in the Fischer-Tropsch catalysis, the carbon nanotubes synthesis and for hydrogen storage applications. Furthermore, hybrid artificial solids containing ferromagnetic particles and $\mathrm{MgO}$ might promote a bottom-up approach to spintronic devices different from the standard route of extrapolating the traditional concepts of microelectronics onto the nanoscale, as we are currently demonstrating, for instance in C. MartinezBoubeta et al. App. Phys. Lett. 94, 062507 (2009). 
We wish to confirm that there are no known conflicts of interest associated with this publication and there has been no significant financial support for this work that could have influenced its outcome.

The manuscript has been read and approved by all named authors and that there are no other persons who satisfied the criteria for authorship but are not listed. We further confirm that the order of authors listed in the manuscript has been approved by all of us.

We confirm that we have given due consideration to the protection of intellectual property associated with this work and that there are no impediments to publication, including the timing of publication, with respect to intellectual property. In so doing we confirm that we have followed the regulations of our institutions concerning intellectual property.

We further confirm that any aspect of the work covered in this manuscript that has involved either experimental animals or human patients has been conducted with the ethical approval of all relevant bodies and that such approvals are acknowledged within the manuscript.

Signed by all authors as follows:

[LIST AUTHORS, and SIGNATURES dated on June $20^{\text {th }}$, except otherwise stated] 


\section{AUTHOR DECLARATION}

Prof. B. Martínez, ben.martinez@icmab.es

PhD. LI. Balcells, balcells@icmab.es

PhD. F. Sandiumenge, felip@icmab.es

PhD. A. Calleja acalleja@icmab.es

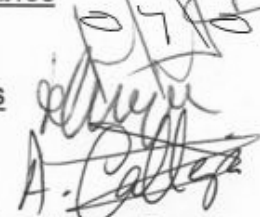

From the Institut de Ciència de Materials de Barcelona (ICMAB-CSIC), Campus UAB, Bellaterra 08193 (Spain)

PhD. R. Cristòfol, rcmfat@iibb.csic.es

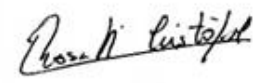

PhD. C. Sanfeliu, cspfat@iibb.csic.es

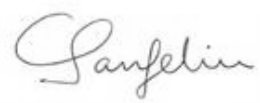

From the Instituto de Investigaciones Biomédicas de Barcelona, CSICIDIBAPS, Barcelona, 08036 (Spain)

MD, Prof. R. Weissleder, nweissleder@mgh.harvard.edu (Uhizt...6/3/is

PhD. E. Rodriguez, evrodriguez@mgh.harvard.edu

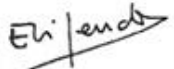

From the Center for Molecular Imaging Research, MGH-Harvard, Boston, MA02114 (US)

PhD. S. Lope-Piedrafita, silvia.lope@uab.es

PhD. Lluis Casas, lluis.casas@uab

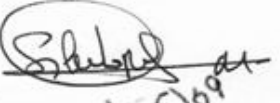

At the Universidad Autónoma de Barcelona, 08193 (Spain)

PhD. K. Simeonidis, ksime@physics.auth.gr

PhD. M. Angelakeris, agelaker@auth.gr

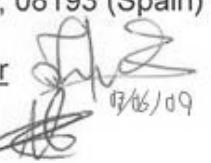

At the Aristotle University of Thessaloniki, Thessaloniki 54124 (Greece)

And

Prof. Claude Monty, claude.monty@promes.cnrs.fr

From the CNRS/Procédés, Matériaux et Energie Solaire, 66120 (France)
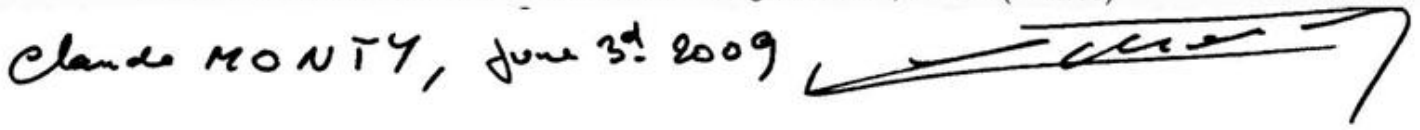

We thank you very much in advance for your kindness. Yours sincerely,

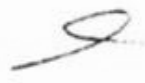

Carlos M. Boubeta 
We suggest the following as the possible reviewers of this work:

\section{Jian-Ping Wang}

Depart. Electrical and Computer

Engineering

Twin Cities Campus

200 Union Street S. E. Minneapolis

MN 55455

jpwang@ece.umn.edu

Phone: 612- 625-9509

Fax: 612-625-4583

\section{Luis Liz-Marzan}

Department of 'Quimica Fisica'

Universidade de Vigo

Office: $26\left(3^{a}\right) /$ Lab 4

Campus Lagoas-Marcosende s/n

36310 Vigo, Pontevedra, Spain

Imarzan@uvigo.es

Phone: (34) 986812298

Fax: (34) 986812556

\section{Hongchen Gu}

Research Institute og Micro/Nano

Science\& Tech.

Nano Biomedical Research Center

800 Dongchuan Rd.

Shangai 200240, China

hcgu@sjtu.edu.cn

Phone: (86) 2134206877

Fax: (86) 2162832374

\section{Manfred Rührig}

Innovative Electronics-Materials and

Microsystems. Siemens AG

CT MM1, P.O. Box 3220

Günther-Scharowsky-Str. 1

Erlangen D-91050

Germany

manfred.ruehrig@siemens.com

Phone: (49) 913117

\section{Linda E. Cammish}

Business Development, Sales and Marketing, nanoTherics Ltd

Tel: (49) 07981112968

Fax: (49) 91317-32469

linda@nanotherics.com 


\section{Self-assembled Multifunctional Fe/MgO Nanospheres for Magnetic Resonance Imaging and Hyperthermia}

By Carlos Martinez-Boubeta ${ }^{a, b,{ }^{*}}$, Lluis Balcells ${ }^{a}$, Rosa Cristòfol ${ }^{c}$, Coral Sanfeliu $^{c}$, Elisenda Rodríguez ${ }^{d}$, Ralph Weissleder ${ }^{d}$, Silvia Lope-Piedrafita ${ }^{e}$, Konstantinos Simeonidis ${ }^{f}$, Makis Angelakeris ${ }^{f}$, Felip Sandiumenge ${ }^{a}$, Alberto Calleja ${ }^{a}$, Lluis Casas ${ }^{g}$, Claude Monty ${ }^{h}$, and Benjamín Martinez ${ }^{a, * *}$

[*] Corresponding author at:

Departament d'Electrònica, Universitat de Barcelona

Martí i Franquès 1, Barcelona 08028 (Spain)

E-mail address: cboubeta@el.ub.es

[**] Corresponding author at:

Institut de Ciència de Materials de Barcelona (ICMAB-CSIC),

Campus UAB, Bellaterra 08193 (Spain)

E-mail address: ben.martinez@icmab.es

${ }^{a}$ ICMAB-CSIC, Campus UAB, Bellaterra 08193, Spain

${ }^{\mathrm{b}} \mathrm{IN}^{2} \mathrm{UB}$, Dept. Electrònica, U.B., 08028, Spain

${ }^{c}$ IIBB, CSIC-IDIBAPS, Barcelona, 08036, Spain

${ }^{\mathrm{d}}$ Center for Molec. Imaging Research, MGH-Harvard Med. School, Boston, MA02114, U.S.

e Servicio de Resonancia Magnética Nuclear, Univ. Autónoma de Barcelona, 08193, Spain

${ }^{\mathrm{f}}$ Unitat de Cristal·lografía i Mineralogia, Dept. Geologia-UAB, 08193, Spain

${ }^{g}$ CNRS/Procédés, Matériaux et Energie Solaire, Font Romeu, 66120, France

${ }^{\mathrm{h}}$ AUTh, Thessaloniki 54124, Greece

\section{ABSTRACT}

A one-step process for the production of nanoparticles presenting advanced magnetic properties can be achieved using vapor condensation. In this paper, we report on the fabrication of Fe particles covered by a uniform MgO epitaxial shell. MgO has a lower surface energy than $\mathrm{Fe}$, which results in a core-shell crystal formation. The particles are proven to be useful as as contrast agents for magnetic resonance diagnosis and heating mediators for cancer therapy through hyperthermia. They also have potential to be used in drug delivery and magnetic-activated cell sorting. 
KEYWORDS: core-shell, vapor condensation, nanoparticles, magnetism, hyperthermia, MRI

\section{INTRODUCTION}

Over the past decades, an increasing number of materials in the nanometer scale have been used in biomedical applications. ${ }^{1}$ In particular, the field of nanomagnetism holds great promise for diagnosis and targeted treatment of human diseases. For instance, combined fluorescence and magnetic resonance imaging (MRI) has become an indispensable tool in cancer research. ${ }^{2,3}$ However, expectations on magnetic approaches have not been fully accomplished, since most of the magnetic nanoparticles (NPs) intended to-date have the disadvantage of been based on superparamagnetic oxides which do not provide a sufficiently large magnetic stray field for high-resolution detection schemes, cell sorting, and magnetically guided drug targeting. ${ }^{4,5,6,7}$

In addition to addressing the drawbacks of toxicity level, size reproducibility and weak magnetic properties, the cost and ease of processing should also be considered as key issues for commercialization. In this context, chemical synthesis is a preferred way to elaborate large quantities of well-controlled NPs. ${ }^{8}$ Nonetheless, the use of precursors in the presence of a large amount of surfactants leads to impurities in the final product that may hampers in-vivo applications. Besides, using the ultrasmall particles achievable within the aforementioned syntheses is not always better when it comes to medicine. Only recently, researchers have demonstrated that spherical particles with a diameter $\sim 50 \mathrm{~nm}$ are taken up by mammalian cells at a faster rate and higher concentration levels compared to other sizes and shapes. This optimal radius means that for the smaller particles to go in, they must be clustered together, ${ }^{9}$ as result of energy penalties arising from the curvature and cell membrane tension. ${ }^{10,11}$ Therefore different coatings on the particles, including inorganic shells or polymers, may lead to different cell uptake and nanocitoxicity, ${ }^{12}$ and influence many vital interactions within the body, including circulation and targeting. ${ }^{13,14,15}$ While a huge 
number of methods have been developed for the scalable synthesis and preparation of inorganic metal and semiconductor nanoparticles, only a few specialized techniques were reported for the preparation of core-shell nanoparticles. ${ }^{16}$

These magnetism-engineered nanoprobes, when conjugated with antibodies, showed enhanced MRI sensitivity for the detection of cancer markers compared with probes currently available. ${ }^{17}$ As well, magnetic particles have been intensively studied as a promising support for tumour therapy schemes, especially when dealing with cancer spreading through the lymph system. Several suggestions for optimizing the use of magnetic NPs in the thermal treatment of cancer tumour are known from literature. For instance, biomimetic material such as bacterial magnetosomes with a mean core diameter of $30 \mathrm{~nm}$ was recently proposed to overcome the drastically decrease in the heating power after immobilisation of particles when penetrating into the tissue. ${ }^{18}$ In addition, superior heating rates are expected if using magnetic materials with higher magnetization and hysteresis losses.

In this work we present an alternative way for synthesizing high quality metallic, soft ferromagnetic Fe nanoparticles with an outer protective $\mathrm{MgO}$ sheath, by using scalable one-step vapour-phase condensation. This technique allows the preparation of large volumes of concentrated nanoparticle dispersions presenting several important advantages over previously described solutions since it achieves a narrow particle size distribution without purification steps, thus reducing processing times. This novel, mechanically stable, biocompatible nanomaterial is shown to be suitable for hyperthermia and magnetic imaging, among other potential biomedical applications. Shortly, in-vivo administration of pristine particles, not chemically modified or functionalized, shown that NPs were apparently cleared from blood stream quickly and distributed throughout most organs within $24 \mathrm{~h}$. The primary accumulation in liver, kidneys and spleen showed a gradual decrease within the 3 weeks monitoring period, for which urinary, and eventually hepatobiliary 
excretion into the intestinal tract, are considered as possible clearance pathways. ${ }^{19,20}$ It should be noted that $\mathrm{Mg}^{2+}$ ions exhibit a biological activity for bone regeneration and it is ubiquitous and essential to all living organism. ${ }^{21}$ Thus, magnesia shells provide non-toxic hydroxyl surface groups that would allow for surface functionalization by covalent linkages of drugs and biomolecules. ${ }^{22}$

\section{METHODS}

II.A.1. Synthesis: Precursors were prepared by mixing Fe (purity $>99.5 \%$ from BASF) and MgO (Alfa Aesar, 99.998\%) powders in the desired proportions. In view of the reactivity of Fe towards oxidation, it was felt to be particularly desirable to ensure the absence of iron oxides by using minute excess Mg (Aldrich, 99.9\%) that provides reducing conditions. ${ }^{23}$ Targets obtained from cold-pressed precursors were vaporized inside a vacuum chamber. During synthesis, the pressure in the chamber was increased by introducing a stream of Ar inert gas. Different sources for evaporation were used. Shortly, when using an environmentally friendly process, solar radiation was channelled to an indoor laboratory via plane mirrors and concentrated onto the target using a parabolic mirror $2 \mathrm{~m}$ in diameter, as described in Ref. 24. The delivered solar power, measured using a calorimeter is around $1500 \mathrm{~W}$ when the incident sun flow is around $1 \mathrm{~kW} \cdot \mathrm{m}^{-2}$. When the energy source was kept and the pressure was reduced, nanoparticles nucleation and growth could be gradually adjusted and size diminished (from about 250 to $20 \mathrm{~nm}$ ) ${ }^{25}$ as a result of the decrease in the mean free path of the vaporized atoms. ${ }^{26}$ Joule heating evaporation was also routinely employed with the same results. Nanomaterial was collected with rates up to $100 \mathrm{mg}$ of powder per hour.

II.A.2. Structure: The detailed structural and morphological characterization of our samples was carried out by means of scanning electron microscopy (SEM), X-ray diffraction (XRD) and high resolution transmission electron microscopy (HRTEM). The precise determination of the crystal phase was performed by means of the fast Fourier transform (FFT) method in each individual nanoparticle. Samples for observation were prepared by drop-casting a dilute ethanol solution of the particles onto carbon-coated copper grids and quickly evaporating the solvent. In order to obtain the HRTEM results we used a field emission gun microscope, JEOL 2010F, which works at $200 \mathrm{kV}$ and has a point-topoint resolution of $0.19 \mathrm{~nm}$. Electron energy loss spectra (EELS) were obtained with an energy resolution of $1.2 \mathrm{eV}$. Energy Dispersive X-ray Analysis (EDX) was used to estimate the powder composition. The thermal stability and hydrogen uptake of the nanoparticles were measured in a thermobalance (TGA) at heating rates of $5{ }^{\circ} \mathrm{C} \cdot \mathrm{min}^{-1}$. 
II.B.1. Magnetic properties: Mössbauer spectra were recorded by using a standard 512 channel spectrometer in transmission geometry with a ${ }^{57} \mathrm{Co}$ in Rh matrix source. For low temperature measurements a He flow cryostat was used. Velocity calibration was done by using a thin $\alpha$-Fe foil. Field and temperature dependent magnetic measurements were performed with a superconducting quantum interferometer device (SQUID) magnetometer in a range of -50 to $50 \mathrm{kOe}$ and 10 to $350 \mathrm{~K}$. By determining the total Fe amount in the SQUID sample using EDX analysis, we found that the saturation magnetization of our crystals was $210 \pm 8$ e.m.u. per metal gram at $300 \mathrm{~K}$. Magnetic measurements and TEM analysis were repeated on some of the samples in order to examine any aging effects, without any degradation over a monitoring period of eighteen months exposure in high humidity-mild temperature Barcelona's ambience conditions.

II.B.2. Hyperthermia studies: Heating experiments for comparing the heating capability of particles were performed in a simple arrangement using a water cooled induction coil of $23 \mathrm{~mm}$ diameter consisting of three turns. The ac field is generated by means of a commercial generator with a power of $4.5 \mathrm{~kW}$. Particles to be investigated were homogeneously dispersed in a relatively large volume of water-based solution which was kept in a thermally isolated glass container inside the ac field coil. We used the grinding technique to produce NPs suspensions on water in the presence of soybean lecithin, allowing an exceptional green process for the production and stabilization of nanoparticles. The dissipated power due to the applied magnetic ac field was determined by measuring the initial slope of the temperature rise by means of a fiber optic temperature probe. As a control, the temperature rise of the water without nanoparticles present was also measured and subtracted from the temperature rise measured for the nanoparticles. It is widely believed that the heating effect is a result of absorbing energy from the alternating magnetic field and transforming it into heat by means of the hysteresis loss during reversal of magnetization. For that reason, and for the sake of comparison, the loss power was also determined from SQUID measured hysteresis losses assuming linear frequency dependence. A descendent loss power was detected while increasing concentration, which may be a signature of dipolar interactions influencing the NP intrinsic magnetic properties. ${ }^{27}$

II.C.1. In vitro Toxicity: Studies of biocompatibility were performed in the $3 \mathrm{~T} 3$ cell line (ATCC, LGC) derived from mouse embryo fibroblasts and in primary neocortical neuron cultures obtained from E17 mouse embryos (CD1, Charles River). Culture medium was DMEM supplemented with 10\% serum (calf serum for 3T3 and fetal bovine serum for neuron cultures) and $50 \mu \mathrm{g} \cdot \mathrm{ml}^{-1}$ gentamicin. Both $3 \mathrm{~T} 3$ and neuron cultures were seeded in 24-well plates, at the densities of $8 \times 10^{3}$ and $2 \times 10^{5}$ cell.cm ${ }^{-2}$, respectively. Fibroblast cells were used at the exponential growth phase after 1 - 2 days in vitro, and neurons when reaching maturity at $6-7$ days in vitro. We changed well media to fresh media containing $\mathrm{Fe} / \mathrm{MgO}$ nanoparticles at concentrations up to $1 \mathrm{mM}$ total-metal molar concentration $\left(\mathrm{Fe}^{0}\right)$. We carried out the 
experimental incubations at the cell culture conditions of $37^{\circ} \mathrm{C}$ and $5 \% \mathrm{CO}_{2}$ atmosphere, for 3 days. Survival was measured in both cell culture types by a live/death stain assay (double staining with fluorescein diacetate/propidium iodide), as described elsewhere, ${ }^{28}$ or the trypan blue exclusion assay. No cytotoxic effects were detected either with fluorescein diacetate/propidium iodide (Fig. 4a and c) or with trypan blue staining. Additionally, 24h proliferation rate was determined in $3 \mathrm{~T} 3$ by cell counting in a hemocytometer (Fig. 2b). Data show the average \pm standard deviation of triplicate assays.

II.C.2. In vivo Toxicity: We maintained 5 female and 5 male CD1 mice $(25-35 \mathrm{~g})$ in accordance with the guidelines and under the approval of CEEA animal care committee of Universitat de Barcelona, Spain. Mice were injected through the vein tail with a solution (10 $\mu$ l per animal gram) of $\mathrm{Fe} / \mathrm{MgO}$ nanoparticles dispersed in PBS at the concentration of $1 \mathrm{mM} \mathrm{Fe}^{0}$. The mice did not show symptoms of discomfort after dosing. Animals were regularly monitored for three weeks, sacrificed and autopsied. No changes in body weight and behaviour or macroscopic alterations of internal organs were detected.

II.D.1. Relaxivity measurements: $\mathrm{T}_{2}$ relaxation times were measured using an inversion-recovery pulse sequence with a Bruker Minispec (Bruker Analytics, North Bellirica, MA) at $37^{\circ} \mathrm{C}$ and $0.47 \mathrm{~T}(20 \mathrm{MHz})$.

II.D.2. MRI imaging and biodistribution: MRI was performed on a 7 Tesla Bruker BioSpec to obtain $\mathrm{T}_{2}$ maps using a MSME sequence with the following parameter: TE $=15.20-242.92 \mathrm{~ms}(16$ echos $), \mathrm{TR}=3573 \mathrm{~ms}$, acquiring 13 contiguous $1.5 \mathrm{~mm}$ slices with a $7.99 \times 3.99 \mathrm{~cm}^{2}$ field of view $(\mathrm{FOV})$, and acquisition matrix $=512 \times 256 \mathrm{MRI} \mathrm{T}_{2}$ weighted images of mice under ketamine anaesthesia were acquired at room temperature. Theoretical considerations predict linear correlations between the proton transverse relaxation rate and concentration of magnetic ions [29]. Here the challenge was to develop a method that provides quantitative information. Therefore, for ex vivo magnetic particle quantization, the animals were sacrificed. The livers, spleen and kidneys were eviscerated, placed in individual tubs $(\sim$ $0.15 \mathrm{~cm}^{3}$ ) and frozen for vibrating sample magnetometer analysis. Thanks to the strong ferromagnetic behaviour, NPs could be easily distinguished from the blood pool. Kidney and spleen magnetic signals were at least an order of magnitude lower than the signal measured from the liver, demonstrating primarily hepatic accumulation. 


\section{III.RESULTS AND DISCUSSION}

A general view of the obtained samples is shown in the electron microscopy image of Fig. 1, here displaying nearly spherical nanoparticles with a primary diameter of about $75 \mathrm{~nm}$ and $16 \%$ standard deviation, in good agreement with the log-normal distribution of particle size expected based on modelling results on the Brownian aggregation of atoms within the free molecular regime. ${ }^{30}$ High-resolution transmission electron microscopy (TEM) and dark-field imaging clearly showed a $\mathrm{MgO}$ layer overcoating a crystalline body-centered-cubic Fe core. Powder X-ray diffraction (XRD) patterns could be indexed to $\mathrm{MgO}$ and Fe crystalline phases (Fig. 1d) with no evidences for the presence of iron oxides, despite the broadening of the diffraction profile at low angles due to the presence of $\mathrm{Mg}(\mathrm{OH})_{2}$ during the passivation process in air. Due to the $\mathrm{Mg}-\mathrm{MgO}$ excess in the initial batch composition, we also observed that a substantial fraction of the sample is in the form of $\mathrm{MgO}$ particles with cube morphology confined only by the $6\{100\}$ planes with the lowest surface energy (Fig. 1f). Therefore, the Fe-core nucleation and MgO-shell growth prove to be differentiated. Assuming that the $\mathrm{MgO}$ self-assemble on the Fe surface because of differences in surface energy, interfacial stress probably modifies the radial growth of the shell and limits the $\mathrm{MgO}$ thickness. $^{31}$

Additional information about the nature of $\mathrm{Fe} / \mathrm{MgO}$ interface was obtained by using Mössbauer spectroscopy (Fig. 2a). Results (Table 1) indicate the ferromagnetic character of the NPs. The observed intensity ratio 3:2:1 between the sextet lines and the value of the hyperfine magnetic field $\left(\mathrm{H}_{\mathrm{i}} \approx 330 \mathrm{kOe}\right)$ indicates that the core of the particles is integrated by $\alpha-\mathrm{Fe}$. On the other hand, the hyperfine parameters of the doublet, isomer shift (IS) and quadrupolar splitting $(\Delta)$, exhibit typical values of $\mathrm{Fe}^{3+}$ cations in octahedral coordination. ${ }^{32,33} \mathrm{We}$ suggest that, for charge compensation at the interface, the $\mathrm{Fe}^{3+}$ submonolayer formation involves $\mathrm{Mg}^{2+}$ vacancies originating from the $\mathrm{Mg}(\mathrm{OH})_{2}$ passivation. $^{34}$ 
As expected, the resulting powders are strongly attracted to a permanent magnet (inset Fig. $2 b$ ), therefore can be employed to separate active nanoparticles in a reaction medium. Magnetization measurements (Fig. 2c) indicate that blocking temperature is well above $300 \mathrm{~K}$. By determining the total Fe amount using energy dispersive X-ray analysis, we found the saturation magnetization $\left(\mathrm{M}_{\mathrm{S}}\right)$ values $\left(\mathrm{M}_{\mathrm{S}} \approx 217\right.$ e.m.u.g ${ }^{-1}$ at $10 \mathrm{~K}$ and $\mathrm{M}_{\mathrm{S}} \approx 210$ e.m.u.g ${ }^{-1}$ at $\left.300 \mathrm{~K}\right)$ very close to that of pure bcc iron (bulk Fe $\approx 223$ e.m.u.g ${ }^{-1}$ ). The inset in Fig. 2c shows a magnification of the low field regime making evident that samples have a coercivity $\left(\mathrm{H}_{\mathrm{C}}\right)$ of about 180 Oe at $300 \mathrm{~K}$, close to the value calculated for magnetization reversal of spherical single domain Fe grains. ${ }^{35}$ Importantly, the very low remanence, compared to an assembly of randomly oriented non-interacting nanoparticles, ${ }^{36}$ explains the organization of chains of nanospheres by magnetic flux closure states. ${ }^{37}$

Interestingly, magnetostatic interactions of the particles moments may alter the frequency dependence of the hyperthermia response. ${ }^{18,37}$ We explored the potential use of the ferromagnetic properties of the $\mathrm{Fe} / \mathrm{MgO}$ particles for cancer therapy by carrying out heating experiments. We found that under field amplitude $\mathrm{H}=250$ Oe and frequency $f=765 \mathrm{kHz}$ nanoparticles solutions exhibited significant temperature increases over time (Fig. 3). A very high specific absorption rate (SAR) in the order of $450 \mathrm{~W} \cdot \mathrm{g}^{-1}$ (per Fe gram) was found (Fig. 3 b), close to the losses power estimated theoretically to be proportional to the square of the moment. ${ }^{18}$ Typically, ferrofluid samples investigated in literature show SAR values in the order of $100 \mathrm{~W} \cdot \mathrm{g}^{-1}$. This demonstrated $\mathrm{Fe} / \mathrm{MgO}$ NPs as high performance therapy vectors capable of induce heat at lower doses than existing materials.

To test the efficacy of our nanomaterial as imaging agent for MRI, we measured longitudinal $\left(T_{1}\right)$ and transverse $\left(T_{2}\right)$ relaxation times of protons in water solutions in a $0.47 \mathrm{~T}$ magnetic field, derived $r_{1}$ and $r_{2}$ relaxivities and compared them (Table 2) with commercial MRI contrast agents and data from literature. ${ }^{15,17,38} \mathrm{~A}$ remarkable result was that $\mathrm{Fe} / \mathrm{MgO} \mathrm{NPs}$, as a consequence of the large 
magnetic nucleus, exhibited quite elevated $r_{2}$ values $\left(152 \mathrm{mM}^{-1} \cdot \mathrm{s}^{-1}\right)$ despite not being fully saturated. Therefore, even higher effects on relaxation times are expected on clinical equipments. Also important was that our NPs revealed a very high $r_{2} / r_{1}$ ratio making them affording effective as $T_{2}$ negative-contrast agents. ${ }^{39,40}$

In terms of biocompatibility, our in vitro toxicity assays on two different cell lines shown no deleterious biological properties (Fig. 4). These results were expected considering the antiseptic properties of $\mathrm{MgO}$ by virtue of its basicity. ${ }^{22}$ In preliminary in vivo animal experiments, we intravenously injected $10 \mu \mathrm{l.g}^{-1}$ of saline solution at $1 \mathrm{mM} \mathrm{Fe}^{0}$ molar concentration into mice (total iron dose about $0.6 \mathrm{mg} \cdot \mathrm{kg}^{-1}$ ), and observed no apparent acute toxicity or side-effects health problems over a monitoring period of 3 weeks, though of fear of raising the likelihood of blood vessel blockage due to particles-cluster formation by magnetic interaction. We suggest that hydrodynamic forces together with the gathering of NPs into elongated assemblies may improve the ability of the active cores to circulate. ${ }^{38}$

To address the biodistribution, animals were imaged using MRI (Fig. 5). We distinguished intensity changes in $\mathrm{T}_{2}$-weighted MR images before injection, as well as 1 day and three weeks after injection. The distribution of particles in the mouse $24 \mathrm{~h}$ post injection is similar to that reported previously for iron oxides particles with similar physical characteristics. ${ }^{41}$ This particles display a tendency to undergo phagocytic system clearance in the liver, spleen and kidneys. Significantly, the values measured for MRI are consistent with the magnetic data analysis of excised organs. A significant fraction of the iron was shown to enter the liver, as can be detected qualitatively in $\mathrm{T}_{2^{-}}$ weighted images by the liver hypo-intensity at $24 \mathrm{~h}$ and 21 days after contrast injection. Accordingly, $T_{2}$ maps show decrease $T_{2}$ values in the liver after contrast administration $\left(T_{2}=15.12 \mathrm{~ms}\right.$ before injection, and equals $10.59 \mathrm{~ms}$ at $24 \mathrm{~h}$ after injection). ${ }^{42}$ 


\section{IV.CONCLUSIONS}

A remarkable result was that the hysteresis cycle taken from tissue several days after injection resembled that of the NPs powder, demonstrating the particle in vivo solidity. Even in case the magnetic nanoparticles start to break down, any $\mathrm{Fe}$ and $\mathrm{Mg}$ amount will be diluted and regulated within the body. Given that a clinical dose would likely include a few milligrams of Fe per kilogram body mass, the prospect of iron overload is highly unlikely. ${ }^{43}$ This result achieved a major goal for MRI contrast-agent and hyperthermia development, from which haemosiderin deposits, and eventually organ disfunction, could be minimized. Although our studies may be considered as a first proof-of-principle approach, further work is still needed to investigate the biocompability of the nanocrystals in various in vivo applications.

\section{Acknowledgments}

We acknowledge financial support from Spanish MEC (MAT2006-13572-C02-01 and SAF2006-13092-C02-02), CONSOLIDER (CSD2007-00041), ISCIII (RD06/0013/1004), FEDER program and Generalitat de Catalunya (2005SGR-00509). This work was partially funded by E. C. project SOLFACE in the frame of the 6ePCRDT program. Authors acknowledge the Servei de Resonancia Magnètica Experimental from IDIBAPS (Barcelona) for MRI measurements. Boubeta thanks the Spanish MEC for the financial support through the Juan de la Cierva \& Ramón y Cajal programs. Boubeta also wish to thank Prof. B. Hjörvarsson for stimulating discussions on hydrogen uptake. E. R. kindly acknowledges the EC-Marie Curie grant OIF (Molecular Imaging 39693). 


\section{References}

[1] Gilchrist RK, Medal R, Shorey WD, Hanselman RC, Parrott JC and Taylor CB, Selective Inductive Heating of Lymph Nodes. Ann Surg 1957;146:596-606.

[2] Weissleder R and Pittet MJ, Imaging in the era of molecular oncology. Nature 2008;452:580-9.

[3] Bruns OT, Ittrich H, Peldschus K, Kaul MG, Tromsdorf UI, and Lauterwasser J et al., Real-time magnetic resonance imaging and quantification of lipoprotein metabolism in vivo using nanocrystals. Nat Nanotech 2009; 4:193-201.

[4] Ito A, Shinkai M, Honda H and Kobayashi T, Medical application of functionalized magnetic nanoparticles. J Biosci Bioeng 2005;100:1-11.

[5] Kim S and Bae S, Optimizing the geometry of an in vitro tunneling magnetoresistance biosensor using an immobilized ferrimagnetic nanoparticle agent. J Appl Phys 2008;104:113911-8.

[6] Neuberger T, Schöpf B, Hofmann H, Hofmann M and von Rechenberg B, Superparamagnetic nanoparticles for biomedical applications: Possibilities and limitations of a new drug delivery system. J Magn Magn Mater 2005;293:483-96.

[7] Lübbe AS, Bergemann C, Huhnt W, Fricke T, Riess H and Brock JW et al., Cancer Res 1996;56:4694-701.

[8] Park J, An K, Hwang Y, Park JG, Noh HJ and Kim JY et al., Ultra-large-scale syntheses of monodisperse nanocrystals. Nat Mater 2004;3:891-5.

[9] Chithrani BD and Chan WCW, Elucidating the mechanism of cellular uptake and removal of protein-coated gold nanoparticles of different sizes and shapes. Nano Lett 2007;7:1542-50.

[10] Zhang S, Li J, Lykotrafitis G, Bao G and Suresh S, Size-dependent endocytosis of nanoparticles. Adv Mater 2009;21:419-24.

[11] Cross SE, Jin YS, Tondre J, Wong R, Rao JY and Gimzewski JK, AFM-based analysis of human metastatic cancer cells. Nanotechnology 2008;19:384003-8. 
[12] Shaw SY, Westly EC, Pittet MJ, Subramanian A, Schreiber SL and Weissleder R, Perturbational profiling of nanomaterial biologic activity. PNAS 2008;105:7387-92.

[13] Mitragotri S and Lahann J, Physical approaches to biomaterial design. Nat Mater 2009;8:15-23.

[14] Tan W, Wang K, He X, Zhao XJ, Drake T and Wang L et al., Bionanotechnology based on silica nanoparticles. Med Res Rev 2004;24:621-38.

[15] Seo SW, Lee JH, Sun X, Suzuki Y, Mann D and Liu Z et al., FeCo/graphitic-shell nanocrystals as advanced magnetic-resonance-imaging and near-infrared agents. Nat Mater 2006;5:971-6.

[16] Xu YH and Wang JP, FeCo-Au core-shell nanocrystals. Appl Phys Lett 2007;91:233107-3.

[17] Lee JH, Huh YM, Jun YW, Seo JW, Jang JT and Song HT et al., Artificially engineered magnetic nanoparticles for ultra-sensitive molecular imaging. Nat Med 2007;13:95-9.

[18] Hilger I, Hergt R and Kaiser WA, Use of magnetic nanoparticle heating in the treatment of breast cancer. IEE Proc Nanobiotechnol 2005;152:33-9.

[19] Burns AA, Vider J, Ow H, Herz E, Penate-Medina O and Baumgart M et al., Fluorescent silica nanoparticles with efficient urinary excretion for nanomedicine. Nano Lett 2009;9:442-8.

[20] Yamago S, Tokuyama H, Nakamura E, Kikuchi K, Kananishi S and Sueki K et al., In vivo biological behavior of a water-miscible fullerene: ${ }^{14} \mathrm{C}$ labeling, absorption, distribution, excretion and acute toxicity. Chem Biol 1995:2:385-9.

[21] Bertinetti L, Drouet C, Combes C, Rey C, Tampieri A and Coluccia S et al., Surface characteristics of nanocrystalline apatites: effect of $\mathrm{Mg}$ surface enrichment on morphology, surface hydration species, and cationic environments. Langmuir 2009;25:5647-54.

[22] Stoimenov PK, Zaikovski V and Klabunde KJ, Novel halogen and interhalogen adducts of nanoscale magnesium oxide. J Am Chem Soc 2007;125:12907-13.

[23] Oh H, Lee SB, Seo J, Min HG and Kima JS, Chemical structure of the interface between MgO films and Fe(001). Appl Phys Lett 2003;82:361-3. 
[24] Martínez B, Obradors X, Balcells L, Rouanet A and Monty C, Low temperature surface spinglass transition in $\gamma-\mathrm{Fe}_{2} \mathrm{O}_{3}$ nanoparticles. Phys Rev Lett 1998;80:181-4.

[25] Kouam J, Ait-Ahcene T, Plaiasu AG, Abrudeanu M, Motoc A and Beche E et al., Characterization and properties of $\mathrm{ZnO}$ based nanopowders prepared by solar physical vapor deposition. Solar Energy 2008;82:226-38.

[26] Gangopadhyay S, Hadjipanayis GC, Dale B, Sorensen CM, Klabunde KJ and Papaefthymiou V et al., Magnetic properties of ultrafine iron particles. Phys Rev B 1992;45:9778-87.

[27] Lacroix LM, Bel Malaki R, Carrey J, Lachaize S, Respaud M and Goya GF et al., Magnetic hyperthermia in single-domain monodisperse FeCo nanoparticles: Evidences for Stoner-Wohlfarth behavior and large losses. J Appl Phys 2009;105:023911-4.

[28] Rodríguez E, Roig A, Molins E, Arús C, Quintero MR and Cabañas ME et al., In vitro characterization of an $\mathrm{Fe}_{8}$ cluster as potential MRI contrast agent. NMR Biomed 2005;18:300-7.

[29] Pierre TG St, Clarck PR and Chua-anusorn W, Single spin-echo proton transverse relaxometry of iron-loaded liver. NMR Biomed 2004;17:446-58.

[30] Grass RN and Stark WJ, Gas phase synthesis of fcc-cobalt nanoparticles. J Mater Chem 2006;16:1825-30.

[31] Wang H, Upmanyu M and Ciobanu CV, Morphology of epitaxial core-shell nanowires. Nano Lett 2008;8:4305-11.

[32] Bhide VG and Tambe BR, Investigation of the MgO:Fe system using the Mössbauer Effect. J Mat Science 1969;4:955-61.

[33] Marchetti SG, Spretz R, Ulla MA and Lombardo EA, Identification of the species formed in the Fe/MgO system: A Raman and Mössbauer study. Hyperfine Interactions 2000;128:453-66.

[34] Goniakowski J, Bouette-Russo S and Noguera C, Acido-basic properties of simple oxide 
surfaces III; Systematics of $\mathrm{H}^{+}$and $\mathrm{OH}^{-}$adsorption. Surf Sci 1993;284:315-7.

[35] Tamura I, Mössbauer effect in oxidized iron fine particles and explanation of the spectra by magnetic fluctuation. J Magn Magn Mater 1995;145:327-36.

[36] Stoner EC and Wohlfarth EP, A mechanism of magnetic hysteresis in heterogeneous alloys. Philos Trans R Soc London A 1948;240:599-642.

[37] Hÿtch MJ, Dunin-Borkowski RE, Scheinfein MR, Moulin J, Duhamel C and Mazaleyrat F et al., Vortex flux channeling in magnetic nanoparticle chains. Phys Rev Lett 2003;91:257207-4.

[38] Park JH, von Maltzahn G, Zhang L, Schwartz MP, Ruoslahti E and Bhatia SN et al., Magnetic iron oxide nanoworms for tumor targeting and imaging. Adv Mater 2008;20:1630-5.

[39] Yang J, Gunn J, Dave SR, Zhang M, Wang YA and Gao X, Ultrasensitive detection and molecular imaging with magnetic nanoparticles. Analyst 2008;133:154-60

[40] Shi X, Wang SH, Swanson SD, Ge S, Cao Z and Van Antwerp ME et al., Dendrimerfunctionalized shell-crosslinked iron oxide nanoparticles for in-vivo magnetic resonance imaging of tumors. Adv Mater 2008;20:1671-8.

[41] Pardoe H, Clark PR, Pierre TG St, Moroz P and Jones SK, A magnetic resonance imaging based method for measurement of tissue iron concentration in liver arterially embolized with ferrimagnetic particles designed for magnetic hyperthermia treatment of tumors. Mag Res Imag 2003, 21, 483-8.

[42] Leroux JC, Injectable nanocarriers for biodetoxification. Nat Nanotech 2007;2:679-84.

[43] Gould P, Nanomagnetism shows in vivo potential. Nanotoday, 2006;1:34-9. 


\section{Figure Captions}

Figure 1. Particle morphology and size distribution. a) General view of the Fe particles deposited on a carbon-film-covered TEM grid. b) Histogram of particles sizes. The curve corresponds to the log-normal probability distribution. c) Composition distribution across a $\mathrm{Fe} / \mathrm{MgO}$ particle acquired by EDX. Inset shows the electron beam scan. d) Podwer-XRD pattern of as-prepared product, from a mixture of $\mathrm{MgO}, \mathrm{Mg}$ and Fe. Peaks are assigned to face-centredcubic $\mathrm{MgO}$ and body-centred-cubic Fe. e) A high-resolution TEM image showing a core-shell structured nanoparticle. The inset exhibits the FFT spectrum taken from the boxed area of the image across the interface. Spots corresponding to the $\mathrm{MgO}$ viewed along the [111] zone axis (marked with circles) and the (110)Fe (indicated by squares) are shown. These results indicate a tendency towards aligning [011]Fe with $[100] \mathrm{MgO}$, giving rise to a low mismatch orientation similar to the one found in thin films. f) Micrograph of a $\mathrm{MgO}$ cube in the vicinity of magnesiacoated Fe sphere.

Figure 2. Magnetic properties of the $\mathrm{Fe} / \mathrm{MgO}$ nanocrystals. a) Mössbauer spectra registered at $80 \mathrm{~K}$ and room temperature. The solid lines are least-squares fits of the experimental points into the superposition of various iron phases. Spectra components in blue are associated with the interfacial $\mathrm{Fe}^{3+}$ sites. b) Field-cooled and zero-field-cooled magnetization measured with an applied magnetic field of 100 Oe. Inset shows the strong attraction of NPs in water suspension towards a magnet. c) High-field hysteresis loops for $\sim 80 \mathrm{~nm}$ nanocrystals measured after several months exposure in ambient air. Inset: low field region at RT (circles) and $10 \mathrm{~K}$ (squares) after field-cooled experiment at $50 \mathrm{kOe}$. 
Figure 3. a) Heating performance of particles aqueous suspensions under 250 Oe and 765 kHz. b) Field-amplitude dependence of SAR at $21 \mathrm{mM}$ (dots) and $10.5 \mathrm{mM}$ (squares) concentration. Heavy line is the loss power determined from minor hysteresis loops.

Figure 4. In vitro biocompatibility analysis. a) Cytotoxicity test on mouse fibroblast 3T3 cells, by incubation with $\mathrm{Fe} / \mathrm{MgO}$ particles for 3 days. Cells were labeled with fluorescein diacetate (green, that reveals cytoplasmic esterase activity in living cells) and propidium iodide (read, dead cells with disrupted membrane) with no effects up to the maximum concentration tested of $1000 \mu \mathrm{M} \mathrm{Fe} \mathrm{e}^{0}$. Upper row, phase contrast; lower row, living/death stain; magnification: $100 \times$ b) Dose-dependent proliferation assay on $3 \mathrm{~T} 3$ cells, by monitoring the $24 \mathrm{~h}$ cell growth. Normal proliferation rates were obtained at all concentrations tested. c) Cytotoxicity test on primary neuron cultures, by incubation with $\mathrm{Fe} / \mathrm{MgO}$ particles for 3 days. Cells were labeled with fluorescein diacetate and propidium iodide, with no effects at the maximum concentration tested. Upper row, phase contrast; lower row, living/death stain; magnification: $200 \times$.

Figure 5. a) $\mathrm{T}_{2}$-weigthed $M R$ images of mouse body before (left) and after injection of nanoparticles. Due to the negative contrast properties of the solution, the liver appears hypointense in images after contrast injection (see arrow). b). Colour-coded $T_{2}$ maps, from yellow (that is, high $\mathrm{T}_{2}$ ) to green (low $\mathrm{T}_{2}$ ). The large magnetic susceptibility of the nanoparticles distorts the local magnetic field in its vicinity, causing the nearby water protons to relax more rapidly, i.e. shortening $T_{2}$ of tissues containing these particles. Thus, as expected, $T_{2}$ maps show a decrease in $T_{2}$ values in the liver after contrast administration. c) Comparison of magnetic signal from targeted liver at the same time points as imagined by MRI. 
Table 1. Hyperfine parameters at 300 and $80 \mathrm{~K}$ for particles produced from an initial $\mathrm{MgO}_{60} \mathrm{Mg}_{15} \mathrm{Fe}_{25}$ (\% wt.) target composition. $\mathrm{H}_{\mathrm{i}}$ : hyperfine magnetic field, in Teslas; IS: isomer shift (all the shifts are referred to $\alpha-\mathrm{Fe}$ at $298 \mathrm{~K}$ ) in $\mathrm{mm} \cdot \mathrm{s}^{-1} ; \Delta$ : quadrupole splitting in $\mathrm{mm} \cdot \mathrm{s}^{-1} ; 2 \varepsilon$ : quadrupolar shift in $\mathrm{mm} . \mathrm{s}^{-1}$; P: relative peak area of iron species. The accuracy of the values obtained from the statistics errors given by the fitting procedure is indicated in parentheses.

\begin{tabular}{|ccccccc|}
\hline $\mathrm{T}(\mathrm{K})$ & fit & $\mathrm{H}_{\mathrm{i}}$ & $\Delta$ & $2 \varepsilon$ & $\mathrm{IS}$ & $\mathrm{P}(\%)$ \\
\hline \hline \multirow{3}{*}{300} & doublet & - & $0.93(8)$ & - & $0.47(5)$ & 12 \\
\hline \hline & sextet & $32.9(2)$ & - & $-0.003(4)$ & $0.003(2)$ & 88 \\
\hline \hline \multirow{2}{*}{80} & doublet & - & $0.92(9)$ & - & $0.538(8)$ & 7 \\
\hline \hline & sextet & $33.8(1)$ & - & $-0.002(6)$ & $0.118(4)$ & 93 \\
\hline
\end{tabular}

Table 2. Relaxativities $r_{1}, r_{2}$ and $r_{2} / r_{1}$ ratios for $\mathrm{Fe} / \mathrm{MgO}$ nanoparticles compared to reported values in literature. The unusually $\mathrm{r}_{2} / \mathrm{r}_{1}$ higher than conventional Gd and iron-oxide based contrast agents may open new possibilities in negative-contrast MRI.

\begin{tabular}{|llllc|}
\hline sample & Applied field $(\mathrm{T})$ & $\mathrm{r}_{1}\left(\mathrm{mM}^{-1} . \mathrm{s}^{-1}\right)$ & $\mathrm{r}_{2}\left(\mathrm{mM}^{-1} \cdot \mathrm{s}^{-1}\right)$ & $\mathrm{r}_{2} / \mathrm{r}_{1}$ \\
\hline \hline $80 \mathrm{~nm} \mathrm{Fe} / \mathrm{MgO}$ & 0.47 & 1.15 & 152 & 132 \\
\hline \hline $7 \mathrm{~nm} \mathrm{FeCo}[15]$ & 1.5 & 70 & 644 & 9.2 \\
\hline \hline Feridex® [15] & 1.5 & 10 & 104 & 10.4 \\
\hline \hline Magnevist ${ }^{\circledR}[15]$ & 1.5 & 4.6 & 4.5 & 1 \\
\hline \hline $12 \mathrm{~nm} \mathrm{MnFe} \mathrm{O}_{4}[17]$ & 1.5 & - & 358 & - \\
\hline \hline $5 \mathrm{~nm} \mathrm{FeO} \mathrm{x}_{\mathrm{x}}$ chains [38] & 4.7 & - & 116 & - \\
\hline
\end{tabular}



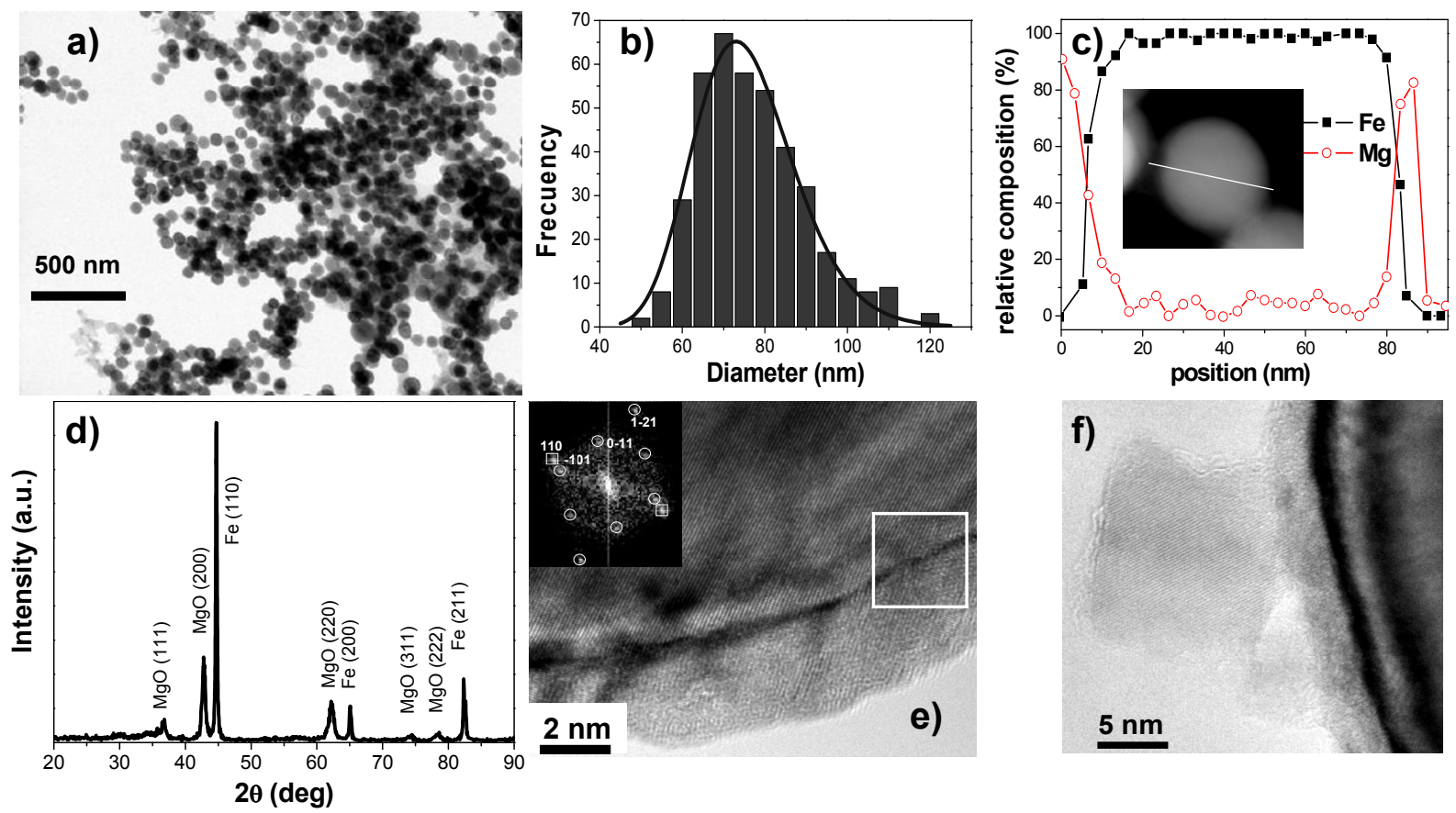

Fig. 1 

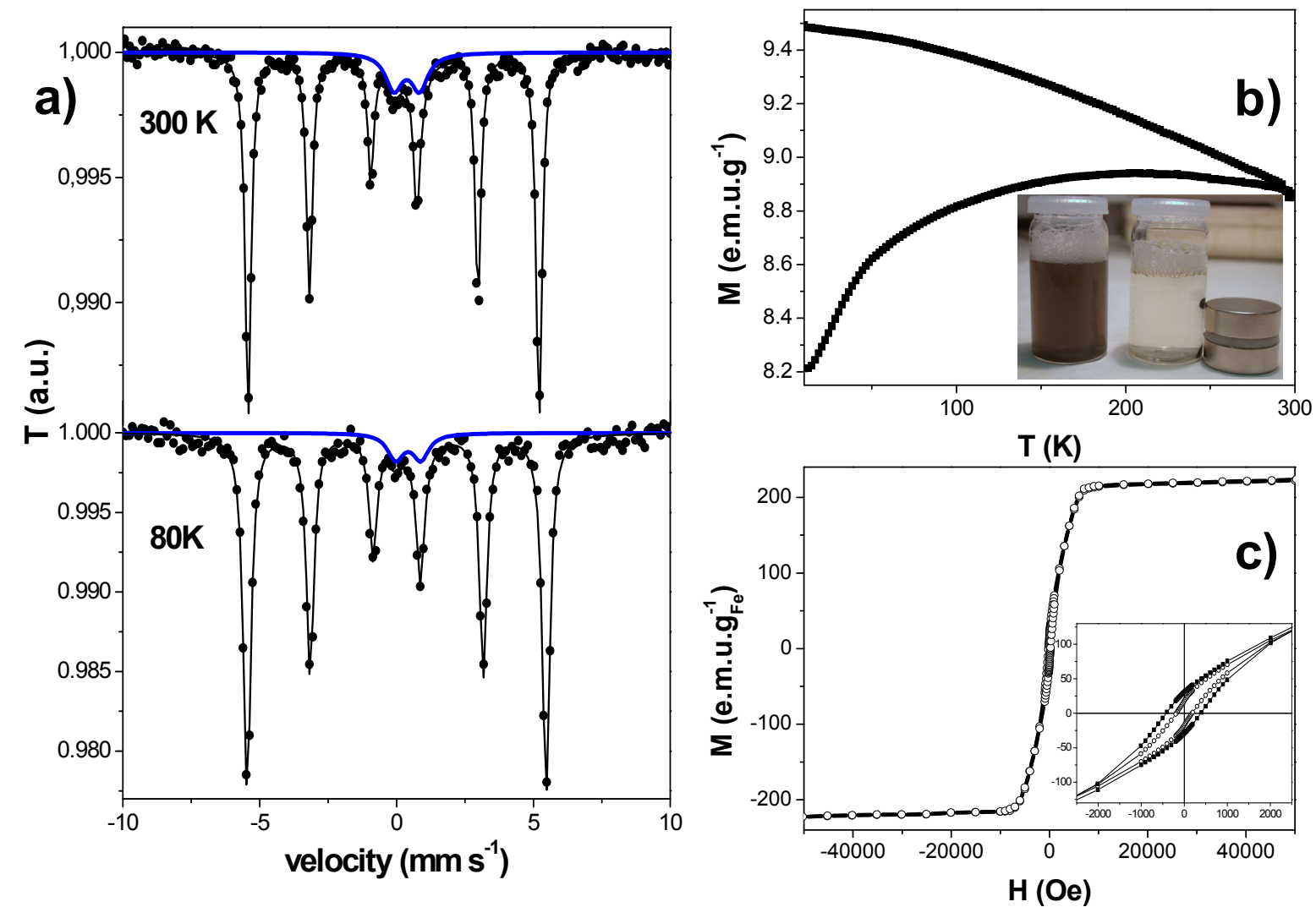

Fig. 2 

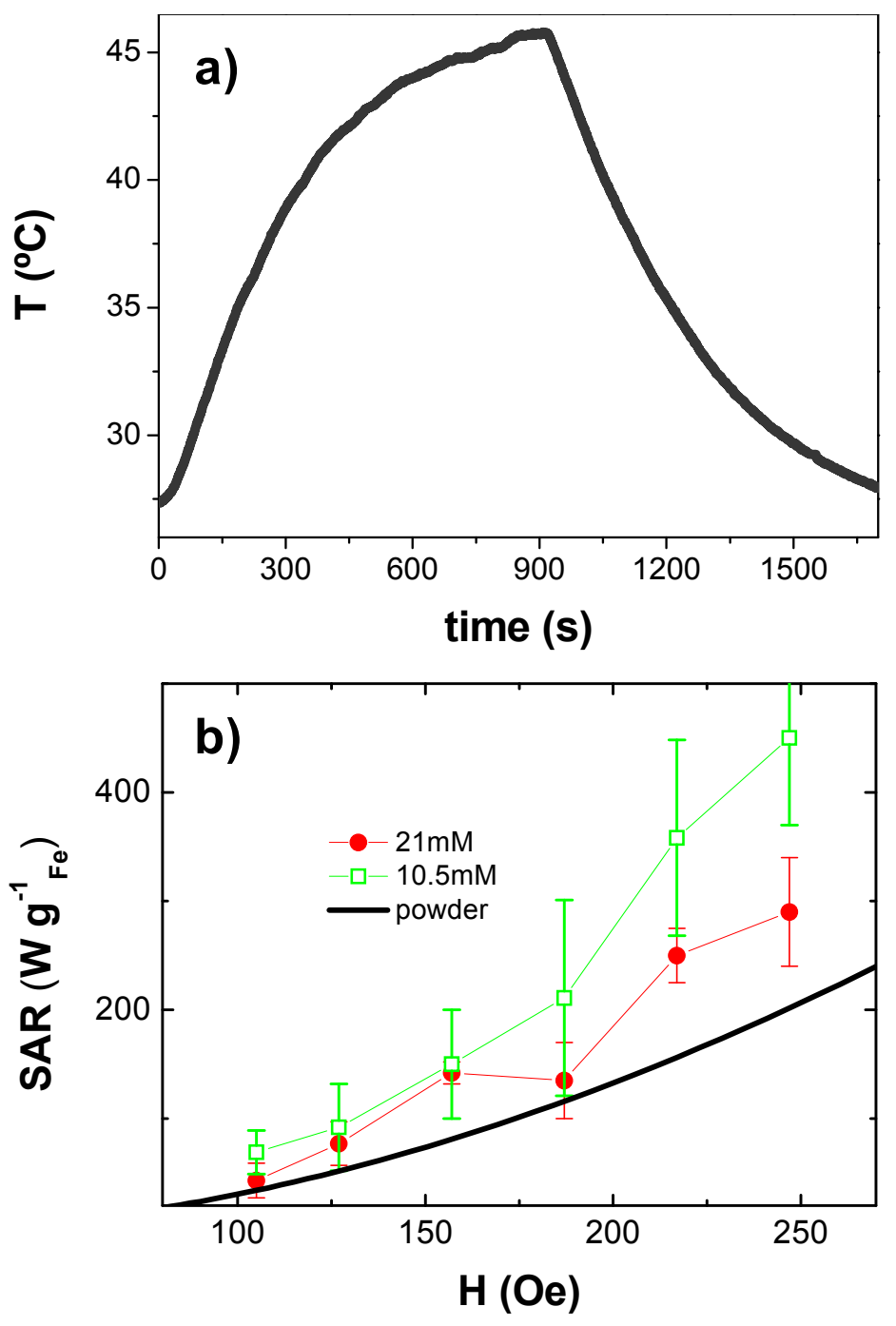

Fig. 3 

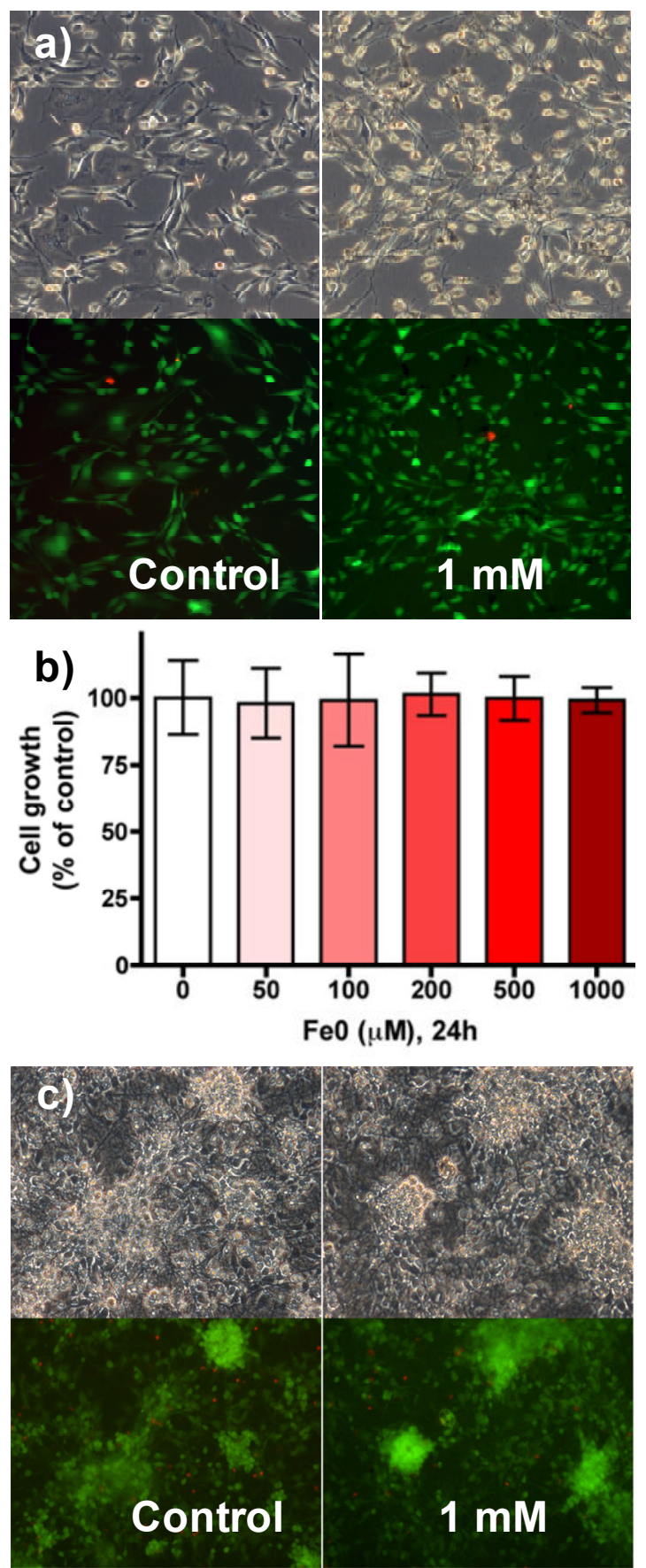

Fig. 4 

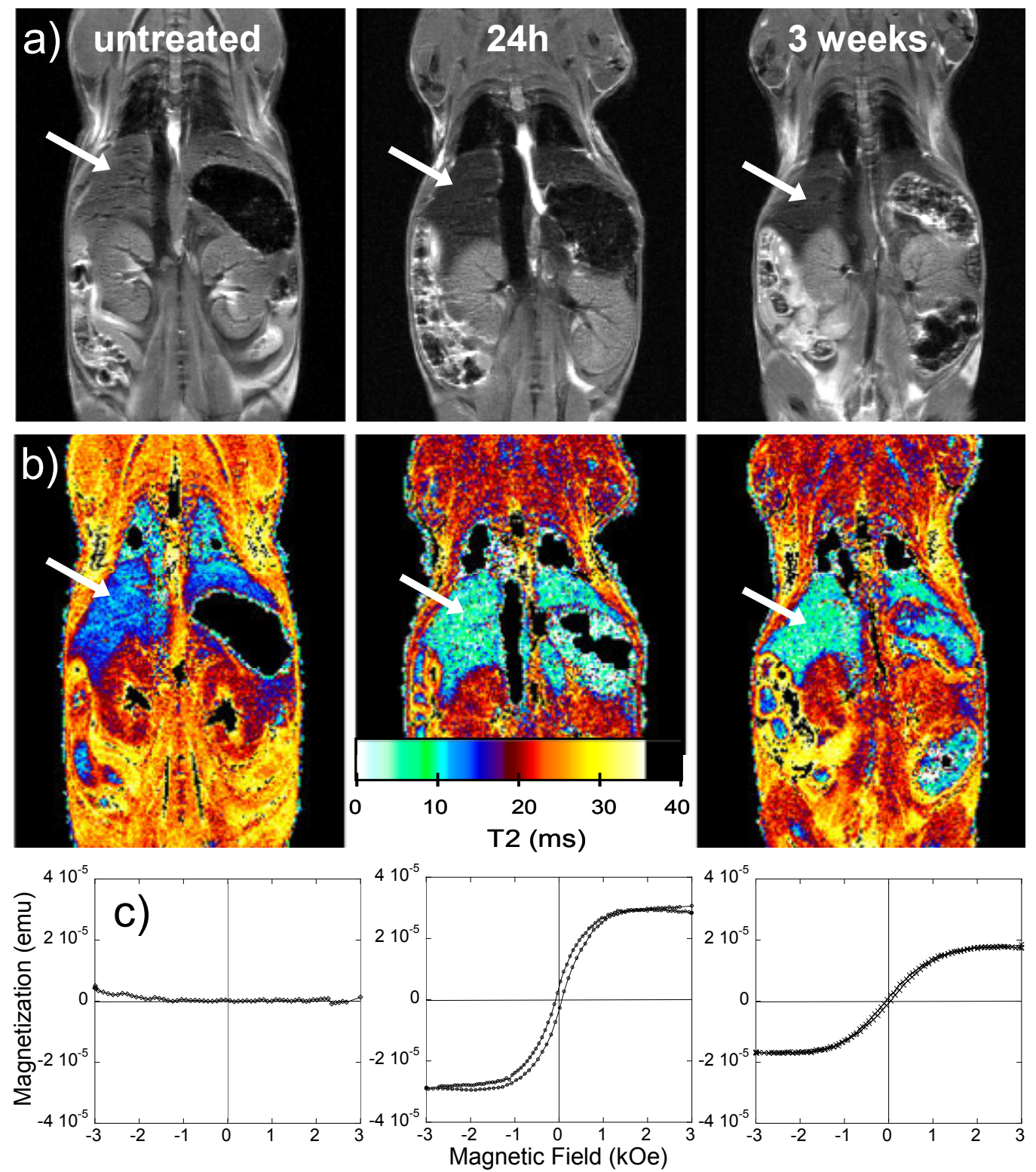

Fig. 5 